\title{
ON GENERALIZED HÖLDER'S INEQUALITY IN WEAK MORREY SPACES
}

\author{
ASYRAF WAJIH ${ }^{1}$ AND HENDRA GUNAWAN ${ }^{2}$ \\ Faculty of Mathematics and Natural Sciences, \\ Institut Teknologi Bandung, Bandung 40132, Indonesia \\ ${ }^{1}$ asyraf.wajih@hotmail.com, ${ }^{2}$ hgunawan@math.itb.ac.id
}

\begin{abstract}
In this paper we reprove generalized Hölder's inequality in weak Morrey spaces. In particular, we get sharper bounds than those in [2]. The bounds are obtained through the relation of weak Morrey spaces and weak Lebesgue spaces.

Key words and phrases: generalized Hölder's inequality, weak Lebesgue spaces, weak Morrey spaces.
\end{abstract}

\section{INTRODUCTION}

Hölder's inequality is one of the classic inequality which is proved by L.J. Rogers in 1888 and reproved by O. Hölder in 1889. Many researchers have obtained new results related to Hölder's inequality. Recently, Ifronika et al. [2] obtained sufficient and necessary conditions for generalized Hölder's inequality in generalized Morrey spaces. Furthermore, they also get similar results for weak Morrey spaces and generalized weak Morrey spaces. In this paper, we present new bounds for generalized Hölder's inequality in weak Morrey spaces which are sharper than those in $[2]$.

For $1 \leq p \leq q<\infty$, the weak Morrey space $w \mathcal{M}_{q}^{p}\left(\mathbb{R}^{n}\right)$ is the set of all measurable functions $f: \mathbb{R}^{n} \rightarrow \mathbb{R}$ such that

$$
\|f\|_{w \mathcal{M}_{q}^{p}}=\sup _{B=B(a, r), \gamma>0}|B|^{\frac{1}{q}-\frac{1}{p}} \gamma|\{x \in B:|f(x)|>\gamma\}|<\infty .
$$

Here $|B|$ denotes the Lebesgue measure of open ball $B=B(a, r)$ centered at $a$ with radius $r$. Note that $\|\cdot\|_{w \mathcal{M}_{q}^{p}}$ defines a quasinorm on $w \mathcal{M}_{q}^{p}\left(\mathbb{R}^{n}\right)$. Furthermore, if $p=q$, then $w \mathcal{M}_{q}^{p}\left(\mathbb{R}^{n}\right)=L^{p, \infty}\left(\mathbb{R}^{n}\right)$. Thus, $w \mathcal{M}_{q}^{p}\left(\mathbb{R}^{n}\right)$ can be considered as generalization of weak Lebesgue space $L^{p, \infty}\left(\mathbb{R}^{n}\right)$. Consequently, the quasinorm

2020 Mathematics Subject Classification: 10406, 2183, 11100 Received: 08-10-2018, accepted: 19-12-2018. 
$\|\cdot\|_{w \mathcal{M}_{q}^{p}}$ can be rewritten as

$$
\|\cdot\|_{w \mathcal{M}_{q}^{p}}=\sup _{B=B(a, r)}|B|^{\frac{1}{q}-\frac{1}{p}}\|\cdot\|_{L^{p, \infty}(B)},
$$

where $\|\cdot\|_{L^{p, \infty}(B)}$ is a quasinorm on weak Lebesgue spaces $L^{p, \infty}(B)$.

From [1], we have the inclusion relation between weak Morrey spaces $w \mathcal{M}_{q}^{p_{1}}\left(\mathbb{R}^{n}\right)$ and $w \mathcal{M}_{q}^{p_{2}}\left(\mathbb{R}^{n}\right)$ for $1 \leq p_{1} \leq p_{2} \leq q<\infty$, as stated in the following theorem.

Theorem 1.1. [1] If $1 \leq p_{1} \leq p_{2} \leq q<\infty$, then $w \mathcal{M}_{q}^{p_{2}}\left(\mathbb{R}^{n}\right) \subseteq w \mathcal{M}_{q}^{p_{1}}\left(\mathbb{R}^{n}\right)$ with

$$
\|f\|_{w \mathcal{M}_{q}^{p_{1}}} \leq\|f\|_{w \mathcal{M}_{q}^{p_{2}}}
$$

for every $f \in w \mathcal{M}_{q}^{p_{2}}\left(\mathbb{R}^{n}\right)$.

\section{MAIN RESULTS}

Let us first state sufficient and necessary conditions for generalized Hölder's inequality in weak Morrey spaces [2].

Theorem 2.1. [2] Let $m \geq 2$. If $1 \leq p \leq q<\infty$ and $1 \leq p_{i} \leq q_{i}<\infty$, $i=1,2, \ldots, m$, then the following statements are equivalent:

(i) $\sum_{i=1}^{m} \frac{1}{p_{i}} \leq \frac{1}{p}$ and $\sum_{i=1}^{m} \frac{1}{q_{i}}=\frac{1}{q}$.

(ii) $\left\|\prod_{i=1}^{m} f_{i}\right\|_{w \mathcal{M}_{q}^{p}} \leq m \prod_{i=1}^{m}\left\|f_{i}\right\|_{w \mathcal{M}_{q_{i}}^{p_{i}}}$, for every $f_{i} \in w \mathcal{M}_{q_{i}}^{p_{i}}\left(\mathbb{R}^{n}\right), i=1,2, \ldots, m$.

Now we will refine the statement of Theorem 2.1, particularly the part which states that (i) implies (ii). Precisely, we will replace the bound $m$ with a smaller constant.

Theorem 2.2. Let $m \geq 2,1 \leq p \leq q<\infty$, and $1 \leq p_{i} \leq q_{i}<\infty, i=1,2, \ldots, m$. If $\frac{1}{p^{*}}=\sum_{i=1}^{m} \frac{1}{p_{i}} \leq \frac{1}{p}$ and $\sum_{i=1}^{m} \frac{1}{q_{i}}=\frac{1}{q}$, then for every $f_{i} \in w \mathcal{M}_{q_{i}}^{p_{i}}\left(\mathbb{R}^{n}\right)$ we have

$$
\left\|\prod_{i=1}^{m} f_{i}\right\|_{w \mathcal{M}_{q}^{p}} \leq \prod_{i=1}^{m}\left(\frac{p_{i}}{p^{*}}\right)^{\frac{1}{p_{i}}}\left\|f_{i}\right\|_{w \mathcal{M}_{q_{i}}^{p_{i}}}
$$

Proof. Let $f_{i} \in w \mathcal{M}_{q_{i}}^{p_{i}}$ for $i \in\{1,2, \ldots, m\}, B=B(a, r) \subseteq \mathbb{R}^{n}$, and $\theta>0$. Suppose that $s_{0}=\theta$ and $s_{m}=1$. Note that for every $s_{1}, s_{2}, \ldots, s_{m-1}>0$ we have

$$
\begin{aligned}
\left|\left\{x \in B:\left|f_{1} f_{2} \cdots f_{m}(x)\right|>\theta\right\}\right| & \leq \sum_{i=1}^{m}\left|\left\{x \in B:\left|f_{i}(x)\right|>\frac{s_{i-1}}{s_{i}}\right\}\right| \\
& \leq \sum_{i=1}^{m}\left\|f_{i}\right\|_{L^{p_{i}, \infty}(B)}^{p_{i}}\left(\frac{s_{i}}{s_{i-1}}\right)^{p_{i}} \\
& =\sum_{i=1}^{m} a_{i} y_{i}^{p_{i}}
\end{aligned}
$$


with

$$
a_{i}=\left\|f_{i}\right\|_{L^{p_{i}, \infty}(B)}^{p_{i}} \quad \text { and } \quad y_{i}=\frac{s_{i}}{s_{i-1}} .
$$

Consequently, for every $y_{1}, \ldots, y_{m}>0$, we have

$$
\left|\left\{x \in B:\left|f_{1} f_{2} \cdots f_{m}(x)\right|>\theta\right\}\right| \leq \sum_{i=1}^{m} a_{i} y_{i}^{p_{i}} .
$$

By choosing

$$
y_{i}=\left(m^{-1} \theta^{-p^{*}} a_{i}^{-1} \prod_{j=1}^{m}\left(\frac{p_{j}}{p^{*}}\right)^{\frac{p^{*}}{p_{j}}} a_{j}^{\frac{p^{*}}{p_{j}}}\right)^{\frac{1}{p_{i}}}
$$

we get

$$
\begin{aligned}
\left|\left\{x \in B:\left|f_{1} f_{2} \cdots f_{m}(x)\right|>\theta\right\}\right| & \leq \sum_{i=1}^{m} a_{i} y_{i}^{p_{i}} \\
& =\sum_{i=1}^{m} a_{i}\left(m^{-1} \theta^{-p^{*}} a_{i}^{-1} \prod_{j=1}^{m}\left(\frac{p_{j}}{p^{*}}\right)^{\frac{p^{*}}{p_{j}}} a_{j}^{\frac{p^{*}}{p_{j}}}\right) \\
& =\theta^{-p^{*}} \prod_{j=1}^{m}\left(\frac{p_{j}}{p^{*}}\right)^{\frac{p^{*}}{p_{j}}}\left\|f_{j}\right\|_{L^{p_{j}, \infty}(B)}^{p^{*}}
\end{aligned}
$$

Moreover,

$$
\begin{aligned}
|B|^{\frac{1}{q}-\frac{1}{p *}} \theta\left|\left\{x \in B:\left|f_{1} f_{2} \cdots f_{m}(x)\right|>\theta\right\}\right|^{\frac{1}{p^{*}}} & \leq|B|^{\frac{1}{q}-\frac{1}{p^{*}}} \prod_{i=1}^{m}\left(\frac{p_{i}}{p^{*}}\right)^{\frac{1}{p_{i}}}\left\|f_{i}\right\|_{L^{p_{i}, \infty}(B)} \\
& =\prod_{i=1}^{m}\left(\frac{p_{i}}{p^{*}}\right)^{\frac{1}{p_{i}}}|B|^{\frac{1}{q_{i}}-\frac{1}{p_{i}}}\left\|f_{i}\right\|_{L^{p_{i}, \infty}(B)} \\
& \leq \prod_{i=1}^{m}\left(\frac{p_{i}}{p^{*}}\right)^{\frac{1}{p_{i}}}\left\|f_{i}\right\|_{w \mathcal{M}_{q_{i}}^{p_{i}} .}
\end{aligned}
$$

By taking the supremum over all open balls $B$ and $\theta>0$, we have

$$
\left\|\prod_{i=1}^{m} f_{i}\right\|_{w \mathcal{M}_{q}^{p *}} \leq \prod_{i=1}^{m}\left(\frac{p_{i}}{p^{*}}\right)^{\frac{1}{p_{i}}}\left\|f_{i}\right\|_{w \mathcal{M}_{q_{i}}^{p_{i}}} .
$$

Hence, by using Theorem 1.1, we get

$$
\left\|\prod_{i=1}^{m} f_{i}\right\|_{w \mathcal{M}_{q}^{p}} \leq\left\|\prod_{i=1}^{m} f_{i}\right\|_{w \mathcal{M}_{q}^{p *}} \leq \prod_{i=1}^{m}\left(\frac{p_{i}}{p^{*}}\right)^{\frac{1}{p_{i}}}\left\|f_{i}\right\|_{w \mathcal{M}_{q_{i}}^{p_{i}}},
$$

which completes the proof.

Next, we prove that the bound in Theorem 2.2 is in general sharper than that in Theorem 2.1. To do so, we need the following theorem. 
Theorem 2.3. [AM-GM Weighted Inequality] Let $m \in \mathbb{N}$. If $x_{i}, w_{i}>0$ for $i=1,2, \ldots, m$, and $w=\sum_{i=1}^{m} w_{i}$. Then

$$
\left(\prod_{i=1}^{m} x_{i}^{w_{i}}\right)^{\frac{1}{w}} \leq \sum_{i=1}^{m} \frac{w_{i} x_{i}}{w}
$$

The equality is attained when $x_{i}=x_{j}$ for every $i, j \in\{1, \ldots, m\}$.

Proof. If $x_{i}=x_{j}$ for every $i, j \in\{1,2, \ldots, m\}$, then equality is clearly attained. Now suppose that $x_{i} \neq x_{j}$ for some $i, j \in\{1, \ldots, m\}$. Since the natural logarithm is a strictly concave function, we can use Jensen inequality to get

$$
\ln \left(\prod_{i=1}^{m} x_{i}^{w_{i}}\right)^{\frac{1}{w}}=\ln \left(\prod_{i=1}^{m} x_{i}^{\frac{w_{i}}{w}}\right)=\sum_{i=1}^{m} \frac{w_{i}}{w} \ln \left(x_{i}\right)<\ln \left(\sum_{i=1}^{m} \frac{w_{i} x_{i}}{w}\right) .
$$

Hence, we have

$$
\left(\prod_{i=1}^{m} x_{i}^{w_{i}}\right)^{\frac{1}{w}}<\sum_{i=1}^{m} \frac{w_{i} x_{i}}{w}
$$

Now we are ready to prove that our bound is sharper than those in [2].

Theorem 2.4. Let $m \in \mathbb{N}, 1 \leq p^{*}<\infty$, and $1<p_{i}<\infty$ for every $i \in\{1, \ldots, m\}$. If $\sum_{i=1}^{m} \frac{1}{p_{i}}=\frac{1}{p^{*}}$, then

$$
\prod_{i=1}^{m}\left(\frac{p_{i}}{p^{*}}\right)^{\frac{1}{p_{i}}} \leq m^{\frac{1}{p^{*}}} \leq m
$$

Proof. Let $\frac{1}{p^{*}}=\sum_{i=1}^{m} \frac{1}{p_{i}}$. By using Theorem 2.3 where $x_{i}=\frac{p_{i}}{p^{*}}, w_{i}=\frac{1}{p_{i}}$, and $w=\frac{1}{p^{*}}$, we get

$$
\left(\prod_{i=1}^{m}\left(\frac{p_{i}}{p^{*}}\right)^{\frac{1}{p_{i}}}\right)^{p^{*}} \leq \sum_{i=1}^{m} \frac{1}{p_{i}} \frac{p_{i}}{p^{*}} p^{*}=m
$$

Hence,

$$
\prod_{i=1}^{m}\left(\frac{p_{i}}{p^{*}}\right)^{\frac{1}{p_{i}}} \leq m^{\frac{1}{p^{*}}} \leq m
$$

as stated. 


\section{CONCLUDING REMARKS}

In this note we already proved that generalized Hölder's inequality in weak Morrey spaces with bound $\prod_{i=1}^{m}\left(\frac{p_{i}}{p^{*}}\right)^{\frac{1}{p_{i}}}$ and this bound is in general sharper than the one obtained in [2].

However, we still do not know whether the bound is really sharp, that is, we still do not know whether there is a function $f_{i} \in w \mathcal{M}_{q_{i}}^{p_{i}}\left(\mathbb{R}^{n}\right), i=1, \ldots, m$ for some $m \geq 2$, such that

$$
\left\|\prod_{i=1}^{m} f_{i}\right\|_{w \mathcal{M}_{q}^{p}}=\prod_{i=1}^{m}\left(\frac{p_{i}}{p^{*}}\right)^{\frac{1}{p_{i}}}\left\|f_{i}\right\|_{w \mathcal{M}_{q_{i}}^{p_{i}}} .
$$

Acknowledgement. We would like to thank the referee for his/her comments on the earlier version of this note.

\section{REFERENCES}

[1] H. Gunawan, D.I. Hakim, K.L. Limanta, and A.A. Masta, "Inclusion properties of generalized Morrey spaces", Math. Nachr., 290:2-3 (2017), 332-340.

[2] Ifronika, M. Idris, A.A. Masta, and H. Gunawan, "Generalized Hölder's inequality in Morrey spaces", Mat. Vesnik., 70:4 (2018), 326-337. 